\title{
An integrated model of impurity migration and wall composition dynamics for tokamaks
}

\author{
K. Schmid ${ }^{*, a}$ M. Reinelt, ${ }^{\text {a }}$ K. Krieger ${ }^{\mathrm{a}}$ \\ ${ }^{a}$ Max-Planck-Institut für Plasmaphysik, Boltzmannstraße 2, D-85748 Garching b. \\ München Germany
}

\begin{abstract}
The prediction of erosion and co-deposition processes for ITER is necessary information for the design and material choice of the first wall. A model has been developed that describes this coupling of local erosion to the global impurity transport and re-deposition processes in a self-consistent way. The erosion and deposition on each surface element of first wall is described by an ordinary differential equation (ODE). The resulting system of ODEs is coupled via the impurity influx, which is derived from the re-distribution of the erosion fluxes through the global impurity transport as calculated by DIVIMP. As a test case, the model is applied to a standard ITER reference discharge calculating the re-distribution of $\mathrm{Be}, \mathrm{C}$ and $\mathrm{W}$ inside the ITER vessel with time.
\end{abstract}

Key words:

PACS: 61.80.Jh, 52.25.Vy, 52.65, 52.40.Hf

\section{Introduction}

In the past 20 years elaborate models based on MD and binary collision Monte Carlo (TRIM like codes) methods have been developed to describe the interaction of energetic particles with the first wall in fusion experiments. Given the usually constant (in composition and energy distribution) incident particle spectra, these codes excel at describing complex phenomena resulting the ejection and deposition of particles from first wall surfaces and the resulting compound formation [1-6]. However, to describe a global erosion deposition balance they lack one crucial component: The back-coupling with the plasma

\footnotetext{
* K. Schmid

Email address: klaus.schmid@ipp.mpg.de (K. Schmid).
} 
impurity transport. In reality erosion at poloidal position A leads to deposition at some other poloidal position B due to a variation in B's incident particle spectrum due to plasma transport of the eroded impurities from A to B. This leads to a complex coupling between the local erosion and deposition phenomena at different poloidal locations. One at first is tempted to directly couple TRIM or MD codes to plasma codes, but this approach has several caveats: To properly simulate the above described coupled simulation one has to calculate the erosion yields and deposition rates due to a complex, ever changing incident spectrum, making it necessary to discretise time in small steps. The problem is that usually the incident flux fractions are in the order of $10^{-4}$, producing a particle spectrum that has to be sampled by at least $10^{7}$ test particles in the simulation to produce a decent representation of the spectrum. While this is in principle possible for TRIM or TRIDYN, for MD this is beyond the current computational possibilities. Further, the discretisation in time is prone to artifacts. MD has another drawback when it comes to cumulative bombardment of surfaces. To simulate, for instance, deposition: Due to computational time limitations, the apparent fluxes in an MD simulation are at least 5-6 orders of magnitude larger that in reality giving rise to all kinds of artifacts [7]. Therefore we propose a rate equation approach that uses parameterized versions of the results of MD or TRIM calculations to determine the proper erosion and reflection rates for a given incident particle system and surface composition. Due to the continuous description of the surface processes in these rate equations one does not run into the problem of having to discretise time or sample distributions. The drawback of course is that one has to use a simpler surface evolution model, but given the uncertainties in the incident fluxes from current SOL codes (e.g. B2/Eirene) this simpler model still is a major step forward compared to the current surface models used in SOL modeling.

The prediction of erosion and co-deposition processes for ITER is a key information for the design and material choice of the first wall. In particular for the current ITER material choice with Be at the main wall, $\mathrm{W}$ at the divertor entrance and dome baffles and CFC at the strike points, it can be expected that co-deposited layers containing $\mathrm{C}, \mathrm{Be}$ and $\mathrm{W}$ are formed. Both the rate at which these layers are formed and their spatial distribution depend critically on the local plasma parameters and on global impurity transport processes: The local plasma parameters thereby define the rate at which deposits or bulk material are (re-) eroded and the global impurity transport defines the incident fluxes of impurities at a given location as a consequence of the corresponding redistribution of eroded material.

The developed model describes this coupling of local erosion and deposition processes to global impurity transport in a self-consistent way: The erosion and deposition on each vertex of the discretised first wall contour is described by an ordinary differential equation (ODE). The resulting system of ODEs is coupled via impurity influx, which is derived from the re-distribution of the local erosion fluxes through the global impurity transport as calculated by 
DIVIMP. To reduce the complexity of the model it is assumed that the time scale of plasma transport is negligible against the time scale of wall evolution. Solving the ODE system one obtains finally the time evolution of the local surface composition on each wall element and the corresponding local erosion flux into the plasma, $\Gamma_{E R O}$. To benchmark the model, Be migration experiments using Be evaporation in JET were used [8]. For the validation process the measured time evolution of Be and $\mathrm{C}$ line emission, corresponding to the respective erosion sources, was compared to predictions of the model [9]. After the successful validation of the model the Be,C, W redistribution in ITER was modeled as a test case for multi-component erosion \& deposition. These processes are critical with respect to formation of low melting Be/W alloys and to growth of in-vessel $\mathrm{T}$ inventories by $\mathrm{T} / \mathrm{Be}$ co-deposition.

\section{$2 \quad$ Modeling}

The aim of the model is not to propose an alternative to TRIM or MD, but to provide an efficient means to use the results from TRIM and MD calculations of PWI modeling in global erosion deposition modeling in SOL impurity transport codes like DIVIMP or in a later stage in B2/E. The model provides means to calculate the time evolution of the erosion and deposition processes occurring due to the global particle transport inside a toroidally symmetric Tokamak fusion experiment. It uses parameterizations of the output of TRIDYN or MD calculations as input to determine the rate coefficients of erosion and deposition. Its surface evolution model is based on the erosion/deposition model presented in [10].

During exposure of a surface to an incident energetic particle flux all of the erosion/deposition processes occur within a thin $(\approx 5$ to $10 \mathrm{~nm})$ near surface region. Therefore the plasma exposed surface is modeled as system of a reaction zone of width $\Delta x$, in which all the erosion and deposition is assumed to take place, located on an infinite bulk. Both the reaction zone and the bulk contain $N$ components and have a homogeneous composition (e.g. 3 for the ITER first wall material choice of Be, $\mathrm{C}$ and $\mathrm{W}$ ) with the amount of each component $e i$ described by its areal density $\delta_{e i}$ in $\# / m^{2}$. (In the subsequent equations indices that refer to an element or component are prefixed by $e$ and indices that refer to a wall element are prefixes by $w$ ). Due to bombardment by the plasma the reaction zone is eroded described by a total sputter yield $Y_{e l, e k}\left(E_{e k}, \delta_{e i \ldots N}\right)$ of component el due to impact of component ek from the plasma. $Y_{e l, e k}\left(E_{e k}, \delta_{e i \ldots N}\right)$ depends on the energy of the impacting element $E_{e k}$ and can depend on the composition $\delta_{e i \ldots N}$ 's of all the other $N$-components in the reaction zone. This composition dependence is important for instance for the erosion of light elements (e.g. C or B) in a heavy matrix (e.g. W). The bombardment also leads to deposition of components described by the influx 
$\Gamma_{e k}\left(\# /\left(m^{2} s\right)\right)$ and reflection yield $R_{e k}\left(E_{e k}, \delta_{e i \ldots N}\right)$ which also depends on the impact energy $E_{e k}$ and can also be a function of the composition of the reaction zone. The key point in the model is to keep the thickness $\Delta x$ of the reaction zone constant by compensating variations due to erosion and deposition by an appropriate material exchange with the bulk. In [10] only two elements of similar number density $\rho\left(\# / \mathrm{m}^{3}\right)$ were taken into account and therefore keeping $\Delta x$ constant was simple. For $\mathrm{N}$ components keeping the $\Delta x$ constant makes the equations very complex due to the composition dependence of the number density $\left(\rho \equiv \rho\left(\delta_{e i}\right)\right)$ in the reaction zone. Therefore in this extension of the basic idea from [10] we keep the total areal density $\delta_{T o t}=\sum_{e i=1}^{N} \delta_{e i}$ constant. Since $\Delta x$ and $\delta_{\text {Tot }}$ are related by $\delta_{T o t}=\rho\left(\delta_{e i}\right) * \Delta x$ the two approaches are essentially identical.

To describe the global erosion/deposition process the poloidal cross section of the first wall is subdivided into $\mathrm{M}$ wall elements, each described according to [10] by a reaction zone on an infinite bulk. Each wall element wr "communicates" with all the other wall elements by its erosion flux which is re-distributed to all other wall elements by the plasma described by a re-distribution matrix $\xi_{e i, w r, w s}$ describing the fraction of particles of component i leaving wall element $w r$ that end up on wall element $w s$. The concept of $\xi_{e i, w r, w s}$ has already been introduced in [11]. The same approach to use DIVIMP to calculate $\xi_{e i, w r, w s}$ was also used here to calculate $\xi_{e i, w r, w s}$ for each component ei. As the local surface compositions change, so do the re-deposited material fluxes onto the first wall. Therefore this approach leads to a coupling between the evolution of incident fluxes and the evolution of the surface composition. To determine $\xi_{\text {ei,wr,ws }}$ test particles are launched homogeneously from each poloidal location in a DIVIMP calculation and their impacts on the other tiles are counted. Since the tiles have different lengths these counts have to be scaled by the length ration of two "communicating" tiles as in eq. 1.

$$
\begin{aligned}
\xi_{e i, w r, w s} & =\frac{N_{w s}^{e i}}{N_{w r}^{e i}} * \frac{l_{w r}}{l_{w s}} \\
N_{w r}^{e i}, N_{w s}^{e i} & =\# \text { of particles launched from wr that impacts in ws } \\
l_{w r}, l_{w s} & =\text { Length of tile wr and ws respectively }
\end{aligned}
$$

Based on the above the change in areal density $\frac{d \delta_{e i, w r}}{d t}$ of component ei on wall element $w r$ can be written as in eq. 2

$$
\text { (2) } \begin{aligned}
\frac{d \delta_{e i, w r}}{d t} & =\Gamma_{e i, w r}^{\mathrm{Dep}}-\Gamma_{e i, w r}^{\mathrm{Ero}}+\Gamma_{e i, w r}^{\mathrm{Bulk}} \\
\Gamma_{e i, w r}^{\text {Dep }} & =\text { Deposition flux of component ei on wall element wr } \\
\Gamma_{e i, w r}^{\text {Ero }} & =\text { Erosion flux of component ei on wall element wr } \\
\Gamma_{e i, w r}^{\text {Bulk }} & =\text { Compensating exchange flux with bulk }
\end{aligned}
$$


The deposition flux of element $e i$ on wall element $w r$ is given by eq. 3

$$
\begin{aligned}
\Gamma_{e i, w r}^{\mathrm{Dep}}= & \Gamma_{e i, w r}^{\mathrm{In}} *\left(1-R_{e i, w r}\left(E_{w i}, \delta_{e j \ldots N}\right)\right) \\
\Gamma_{e i, w r}^{\mathrm{In}}= & \sum_{w s=1}^{M}\left(\Gamma_{e i, w s}^{\mathrm{Ero}}+\Gamma_{e i, w s}^{\mathrm{In}} * R_{e i, w s}\left(E_{w i}, \delta_{e j \ldots N}\right)\right) * \xi_{e i, w r, w s} \\
= & \text { Influx of component ei on wall wr due to } \\
& \text { erosion/reflection on/from the other wall elements } \\
& \text { and plasma transport } \\
R_{e i, w r}= & \text { Reflection yield of element ei on wall tile wr }
\end{aligned}
$$

The erosion flux of element $e i$ on wall element $w r$ is given by eq. 4

$$
\begin{aligned}
\text { (4) } \Gamma_{e i, w r}^{\mathrm{Ero}} & =\sum_{e j=1}^{N} C_{e i, w r} * \Gamma_{e j, w r}^{\mathrm{In}} * Y_{w r, e i, e j}\left(E_{e j}, \delta_{e i \ldots N}\right) \\
Y_{w r, e i, e j}\left(E_{j}, \delta_{e i \ldots N}\right) & =\text { Total sputter yield of component ei by component ej on wall wr } \\
C_{e i, w r} & =\text { Reaction zone concentration of component ei on wall wr }
\end{aligned}
$$

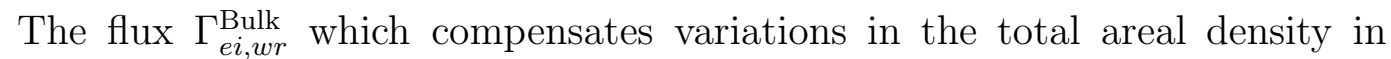
the reaction zone due to erosion and deposition by an appropriate material exchange with the bulk depends on wether the reaction zone is net-depositing or net-eroding. In the net deposition case $\left(\Gamma_{e i, w r}^{\text {Dep }}-\Gamma_{e i, w r}^{\text {Ero }} \geq 0\right)$ material from the reaction zone has to moved to the bulk whereas in the net-erosion case $\left(\Gamma_{e i, w r}^{\text {Dep }}-\Gamma_{e i, w r}^{\text {Ero }} \leq 0\right)$ material from the bulk has to be moved to the reaction

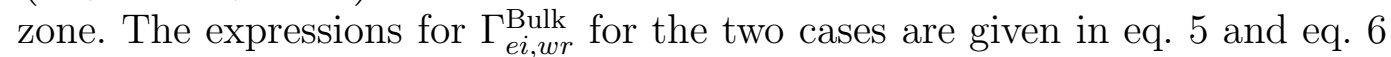
respectively.

$$
\begin{aligned}
\Gamma_{e i, w r}^{\mathrm{Bulk}, \mathrm{Dep}} & =C_{e i, w r} * \sum_{e j=1}^{N} \Gamma_{e i, w r}^{\mathrm{Dep}}-\Gamma_{e i, w r}^{\mathrm{Ero}} \\
C_{e i, w r} & =\text { Reaction zone concentration of component ei on wall wr }
\end{aligned}
$$

(6) $\Gamma_{e i, w r}^{\text {Bulk,Ero }}=C_{e i, w r}^{B u l k} * \sum_{e j=1}^{N} \Gamma_{e i, w r}^{\text {Dep }}-\Gamma_{e i, w r}^{\text {Ero }}$

$$
C_{e i, w r}^{B u l k}=\text { Bulk concentration of component ei on wall wr }
$$

Combining eq. 2 with eq. 5 and eq. 6 the final equation for the areal density change can be written as in eq. 7 


$$
\begin{aligned}
\frac{d \delta_{e i, w r}}{d t}= & \Gamma_{e i, w r}^{\text {Dep }}-\Gamma_{e i, w r}^{\text {Ero }}-\left(1-\operatorname{UStep}\left(\bar{\Gamma}_{w r}^{E r o, D e p}\right)\right) * \Gamma_{e i, w r}^{\text {Bulk,Ero }}- \\
& \operatorname{UStep}\left(\bar{\Gamma}_{w r}^{E r o, D e p}\right) * \Gamma_{e i, w r}^{\text {Bulk,Dep }} \\
\bar{\Gamma}_{w r}^{E r o, D e p}= & \sum_{e j=1}^{N} \Gamma_{e i, w r}^{\text {Dep }}-\Gamma_{e i, w r}^{\text {Ero }} \\
= & \text { Net change in areal density due to erosion \& deposition } \\
= & \text { on wall element wr } \\
\operatorname{UStep}(x)= & \left\{\begin{array}{l}
1 \text { for } \mathrm{x} \geq 0 \\
0 \text { for } \mathrm{x}<0
\end{array}\right.
\end{aligned}
$$

The combination of the algebraic equation system defined in eq. 3 with the differential equation system defined by eq. 7 via $\Gamma_{e i, w r}^{\text {Dep }}$ describes the full time evolution of the composition change on the first wall due to erosion subsequent plasma transport and (re-)deposition. The system contains N-elements $\times \mathrm{M}$-wall tiles differential and $\mathrm{N} \times \mathrm{M}$ algebraic equations which are setup and solved in Mathematica.

The use of the UStep function in eq. 7 switches the compensating flux between the net erosion and the net deposition case and vice versa. Of course since the bulk composition is not altered, a transition in the direction from net deposition to net erosion is not handled correctly, since in reality after the transition the previously deposited material would be eroded. In its current form the model can not handle these transition correctly. The calculations presented later showed that for a constant (only varying impurity fluxes) background plasma these transitions are scarce and only occur in the initial phase of the calculation. If one wants to simulate a varying plasma background one has to daisy-chain several calculations using the end result of the previous calculations as the initial conditions of the next. The transition from net erosion to net deposition is of course handled correctly by the model.

The model distinguishes between accumulating and non accumulating species and between constant influx and varying influx species. The idea is that for instance $\mathrm{D}$ or He are non accumulating species (i.e no equation 7 ) which only enter the model as a species which erode material at the surface. Also the local flux of $\mathrm{D}$ and He are kept constant (i.e no equation 3) making them also constant flux species. To demonstrate the need for an improved surface model in SOL modeling two cases were distinguished with respect to the $\mathrm{C}$ flux: Since C is part of the B2/Eirene plasma solution used for the ITER case calculations, the $\mathrm{C}$ flux was kept constant in one case. In the second case the C flux was calculated self consistently in the model. Since the local C plasma concentrations are tightly coupled to the plasma solution due its radiative plasma cooling properties, dynamic changes in the $\mathrm{C}$ plasma concentration due to changes in the $\mathrm{C}$ erosion source would have a significant impact on the B2/Eirene plasma solution. For constant flux species the ion flux $\Gamma_{e j, w r}^{\mathrm{In}}$ in equation 4 is taken directly from the B2/Eirene background plasma solution 
The erosion flux in eq. 4 is determined by the total sputter yield $Y_{w r, e i, e j}\left(E_{e j}, \delta_{e i \ldots N}\right)$ which in the most general case depends on energy and composition. The energy of the incident particle of type ej on wall tile wk $E_{e j, w k}$ is calculated as in [11] as function of the electron $T_{w k}^{e}$ and ion temperature $T_{w k}^{I}$ on wall tile $\mathrm{k}$ as in eq. 8

$$
\begin{aligned}
E_{e j, w k} & =3 q T_{w k}^{e}+2 T_{w k}^{I} \\
q & =\text { Charge state in which element ej hits wk }
\end{aligned}
$$

Due to the charge state dependence of the energy one would in principle have to extend equation 3 to also include a sum over all charge states. This would, in particular for $\mathrm{W}$, massively increase the number of simultaneous algebraic equations for the fluxes. Therefore the model calculates an average energy (averaged over the charge states) according to eq. 9 .

$$
\begin{aligned}
\left\langle E_{e j, w s}\right\rangle & =\frac{\sum_{w k}^{N} \sum_{q}^{q_{\max , e j}} \Gamma_{e j, w k}^{\mathrm{Dep}} * \tilde{\xi}_{e i, q, w k, w s} * 3 q T_{w s}^{e}+2 T_{w s}^{I}}{\sum_{w k}^{N} \sum_{q}^{q_{\max , e j}} \Gamma_{e j, w k}^{\mathrm{Dep}} * \tilde{\xi}_{e i, q, w k, w s}} \\
q_{m a x, e j} & =\text { Maximum charge state of element ej } \\
\tilde{\xi}_{e i, q, w k, w s} & =\text { Charge state resolved re-distribution matrix from DIVIMP }
\end{aligned}
$$

The charge state resolved re-distribution matrix $\tilde{\xi}_{e i, q, w k, w s}$ describes the fraction of element $\mathrm{i}$ eroded on wall tile wk that ends up on wall tile ws in charge state q. The non charge state resolved re-distribution matrix $\xi_{e i, w k, w s}$ used in eq. 3 is actually derived from $\tilde{\xi}_{e i, q, w k, w s}$ by summing over q. Eq. 9 still depends on $\Gamma_{e j, w k}^{D e p}$ which changes during the course of the calculation (i.e changes with plasma exposure time). By using the fact that $\tilde{\xi}_{e i, q, w k, w s}$ is a rather sparse, almost diagonal matrix eq. 9 can be approximated by eq. 10

$$
\left\langle E_{e j, w s}\right\rangle \approx \frac{\sum_{w k}^{N} \sum_{q}^{q_{\max , e j}} * \tilde{\xi}_{e i, q, w k, w s} * 3 q T_{w s}^{e}+2 T_{w s}^{I}}{\sum_{w k}^{N} \sum_{q}^{q_{\max , e j}} \tilde{\xi}_{e i, q, w k, w s}}
$$

The assumption leading to eq. 10 at first appears rather strong, but a test calculation applying eq. 9 to the $\Gamma_{e j, w k}^{\text {Dep }}(t)$ resulting from a calculation applying eq. 10 show that the variations $\left\langle E_{e j, w k}\right\rangle$ are rather small, mostly in the $\%$ range, justifying the approximation. This now allows to use constant impact energies $E_{e j, w k}$ and thus only the composition dependence of $Y_{w r, e i, e j}$ and $R_{e j, w r}$ needs to be taken into account dynamically during the calculation. The energy dependence can be pre-calculated. For mixtures of low-Z elements like $\mathrm{C}$ and Be the variation of the total sputter yield with composition is weak and can generally be neglected. But for mixtures containing $\mathrm{W}$ the enhanced reflection of projectiles in the near surface region leads to strong enhancement of physical sputtering in the presence of even small amounts of $\mathrm{W}$ as was already explained in [11]. 
The model is very flexible with respect to the applied sputter model and different choices can be implemented. It always includes the effect of dilution since it calculates the erosion flux as the product of the total sputter yield times the surface concentration times the incident flux. To include the additional nonlinear dependence on the surface composition (e.g. the influence of a heavy element like $\mathrm{W}$ in a light Be matrix), the total sputter yield also has a composition dependence. For calculations presented here the total sputter yield $Y_{w r, e i, e j}\left(E_{e j}, \delta_{e i \ldots N}\right)$ required in eq. 4 is described by a scaling law as shown in eq. 11

$$
\begin{aligned}
Y_{w r, e i, e j} & =Y_{e i, e j}\left(E_{e j}\right) *\left(1+\sum_{e k \neq e i}^{N} \delta_{e k, w r} a_{e k}\right) \\
Y_{e i, e j}\left(E_{e j}\right) & =\text { Energy dependence of sputtering of ei by ej } \\
\delta_{e k, w r} & =\text { Areal density of component ek on wall element wr } \\
a_{e k} & =\text { Free parameter describing the composition dependence }
\end{aligned}
$$

The Bohdansky formula [12,13] is used for physical sputtering $Y_{e i, e j}\left(E_{e j}\right)$ of elements which has two free parameters $\left(E_{\text {Thres }}, \mathrm{Q}\right)$ is used. For the composition dependence a linear function is used excluding the terms where $e k=e i$. This avoids quadratic terms $\left(\delta_{e k, w r}^{2}\right)$ in eq. 4 which would have a negative effect on the stability of the solution of the differential algebraic system. To determine the free parameters in eq. 11 the composition and energy parameter range was scanned by a large number TRIDYN [14] calculations. In these calculations the composition of $\mathrm{Be}, \mathrm{C}$ and $\mathrm{W}$ in the target was varied from 0 to 1 and the projectile energy of $\mathrm{Be}, \mathrm{C}, \mathrm{W}, \mathrm{D}$ and $\mathrm{He}$ was varied from $20 \mathrm{eV}$ to $1 \mathrm{keV}$ thus covering the typical parameter range encountered at the first wall of ITER. Then the scaling law in eq. 11 was fitted to the result from the TRIDYN calculations, yielding for each combination of projectile and target $E_{\text {Thres }}, \mathrm{Q}$ and $a_{k}$ values (for clarity the indices relating $E_{\text {Thres }}, \mathrm{Q}$ and $a_{k}$ to a particular projectile and target combination are omitted). In the current calculations chemical erosion of $\mathrm{C}$ is not taken into account. While it would be straight forward to implement in the flexible model, it would probably not have a large impact on the erosion $\mathrm{C}$ : The high temperature at the $\mathrm{C}$ strike points make chemical erosion not the dominant erosion channel and in a material mix with Be and W, C is usually bound in carbide form which is also known [15] to suppress chemical erosion.

In addition to the sputter yield also the composition and energy dependence of the reflection yield for each of the accumulating species (Be, C, W) was described by a scaling law. The form of the scaling law for the reflection yield in eq. 12 is similar except that the energy dependence is now $\propto E^{\alpha}$ which is a reasonable choice for backscattering. 


$$
\begin{aligned}
R_{e j, w r}\left(E_{e j}, \delta_{e i \ldots N}\right) & =\varrho E_{e j}^{\alpha} *\left(1+\sum_{e k}^{N} \delta_{e k, w r} b_{e k}\right) \\
12) \quad & \text { Areal density of component ek on wall element wr } \\
\delta_{e k, w r} & =\text { Free parameter describing the composition dependence } \\
b_{e k} & =\text { Free parameters describing the energy dependence }
\end{aligned}
$$

As for the sputter yield data the reflection yield scaling law was fitted to the result from the TRIDYN calculations yielding for each projectile $\varrho, \alpha$ and $b_{k}$ values (again for clarity the indices relating $\varrho, \alpha$ and $b_{k}$ to a particular projectile and target combination are omitted). The deviations between the reflection yield scaling law and the TRIDYN results are even smaller than for the sputter yield fit.

The above described model can perform similar calculations as presented in [11] but it allows to monitor the full time evolution of the surface composition and impurity fluxes into the plasma, also in contrast to [11] it can handle any number of components. Therefor feeding the calculated time evolution of the erosion fluxes back to impurity transport code allows comparison with spectroscopy data, see for instance [9]. There the model is applied to Be migration experiments performed in JET.

A similar approach was used in [16] by Brooks et al. to describe the mixed material formation in the ITER divertor. They also used the concept of a redeposition matrix to describe the plasmas transport of eroded material which was introduced earlier in [11]. However they did not produce a global self consistent flux balance, they only considered the Be flux variation in the divertor and described the main wall as a constant source similar to what was done in [11]. Also their surface model only considers variations in thickness due to Be deposition. In contrast to [16] our surface model is based on a coupled differential equation of the areal density (i.e composition) evolution of all deposited species. In the presented ITER case three elements (Be, C, W) are considered, but more are possible see [9]). Also our model produces a self consistent global erosion deposition flux balance over the entire poloidal circumference of the first wall for all deposited species. Therefore we think of our model as generalization and refinement of the ideas in $[16,11,10]$.

\section{Results and discussion}

The coupling of the wall tiles in the erosion deposition model is characterized by a re-distribution matrix calculated by DIVIMP as described in [11]: For each wall element a single DIVIMP calculation is performed tracing how material launched at this element is re-distributed around the vessel. As an example the charge state integrated re-distribution matrix for $\mathrm{Be}$ is shown in Fig. 1. It is weakly populated and dominated by its diagonal terms which 
describe local re-deposition. Only in the divertor area the diagonal fans out due to long range transport from the local sources to the divertor. The redistribution matrix for $W$ looks similar for the low charge states $(q<20)$ but for the higher charge states which require deeper SOL penetration, resulting in longer parallel transport, the diagonal generally fans out leading to a broader deposition of higher charge states.

The erosion \& deposition of the first wall elements is described by the composition and energy dependent sputter and reflection yields. As described in section 2 the sputter and reflection yields are described by scaling laws that were fitted to TRIDYN calculations. The quality of the fit is exemplified in Fig. 2 where a comparison of the Be sputter yield by $\mathrm{D}$ and reflection yield of Be with the respective scaling law values is shown. The average deviation between the TRIDYN calculated values and the scaling law values is in the order of $\approx 10 \%$ for the sputter yield and $\approx 20 \%$ for the reflection yield. Given the large uncertainties in the incident particle fluxes from the background plasma solution such deviations in the yields do not affect applicability of the model. The fit parameters for the scaling law for the sputter yield show that the composition dependence is only due to the presence of $\mathrm{W}$. The $a_{e k}$ values (see eq. 11) for Be and $\mathrm{C}$ are essentially zero compared to the $a_{e k}$ value for $\mathrm{W}$. This is not unexpected since the composition dependence of the sputter yield is related to the composition dependence of the reflection yield [11] of the sputtering species and this dependence is weak for elements with low Z. Based on the described model and input parameters the evolution of the plasma facing surface in for an ITER reference discharge (case iter884 [17]) was calculated yielding the time and poloidal position resolved surface compositions and impurity influxes into the plasma. These impurity influxes determine the plasma concentration of for instance $\mathrm{C}$ which due to radiative cooling has a strong influence on the background plasma solution as determined by $\mathrm{B} 2 /$ Eirene. The deposition of Be from the main chamber onto the divertor $\mathrm{C}$ components reduces the $\mathrm{C}$ erosion influx into the plasma [18] and thus the plasma $\mathrm{C}$ concentration essentially invalidating the B2/Eirene plasma solution. To illustrate this and thus show the need for an improved PWI model, in current SOL codes, the $\mathrm{C}$ erosion flux from the inner divertor in ITER is calculated under two scenarios: In the first scenario the $\mathrm{C}$ influx from the plasma is kept constant. This of course is not realistic since in reality changes in the $\mathrm{C}$ erosion source due to deposition of e.g. Be also changes the influx of $\mathrm{C}$, but this is how currently the SOL modeling is performed. In the second scenario the $\mathrm{C}$ influx is variable and is calculated in a self consistent way including all the changes in the erosion sources. The result of these two calculations in depicted in Fig. 3 which shows the erosion fluxes and the Be surface concentration at the inner divertor strike point area. Initially this surface is pure $\mathrm{C}$, but quickly gets covered with Be reducing the $\mathrm{C}$ erosion flux. In the first scenario due to the artificially kept constant, very high $\mathrm{C}$ influx, the surface stays mainly a $\mathrm{C}$ surface. In the second scenario were the $\mathrm{C}$ influx in calculated self consistently, the coverage of the $\mathrm{C}$ by the Be reduces both the $\mathrm{C}$ erosion flux and thus also 
the $\mathrm{C}$ influx significantly. This is turn leads to a reduction of the $\mathrm{C}$ plasma concentration which requires an adjustment of the B2/Eirene solution.

The erosion \& deposition pattern found in the rest of the poloidal circumference is similar to result found in [11] where a full $\mathrm{W}$ divertor and Be main chamber was considered. In [11] Be deposition was only found at the inner baffle and at the dome. In the calculations presented here deposition was also found in the inner divertor strike point area as described above. This difference to the results in [11] is due to the strong re-erosion of Be from the pure W strike points considered in [11].

The results from this ITER test case calculation show the flexibility of the model which, due to its simplicity, can be easily coupled to complex SOL codes. It allows to use the output from more involved PWI codes in SOL modeling.

\section{Conclusions}

A global impurity transport model has been developed that allows to calculate time evolution of surface composition on the first wall of fusion experiments. It can handle any number of chemical elements and wall configurations. It can also be extended to include chemical phase formations and other material erosion channels like sublimation [19]. Using scaling laws that are fitted to TRIDYN based multi component sputter and reflection yield calculations the energy and composition dependence of the total sputter and reflection yield is calculated at each wall element. The code has been applied to the current ITER first wall configuration including $\mathrm{Be}, \mathrm{C}$ and $\mathrm{W}$, calculating the time evolution of the surface composition. The resulting equilibrium values for Be deposition are in line with previous predictions in literature. The calculation also show how erosion and re-deposition may have an impact on background plasma solution through the variation in the $\mathrm{C}$ influx into the plasma due to deposition of $\mathrm{Be}$ on the $\mathrm{C}$ strike points. This show the necessity for a global erosion deposition code that can be coupled to existing plasma codes. 


\section{References}

[1] K. Ohya, Y. Nakayama, Y. Hamada, T. Tanabe, A. Kirschner, V. Philipps, N. Noda, Phys. Scr. T 111 (2004) 138.

[2] K. Inai, K. Ohya, G. Kawamura, Y. Tomita, J. Plasma Fusion Res. 8 (2009) 433.

[3] K. Ohya, Phys. Scr. T 124 (2006) 70.

[4] K. Ohya, Y. Kikuhara, K. Inai, A. Kirschner, D. Borodin, A. Ito, H. Nakamura, T. Tanabe, J. Nucl. Mater. 72 (2009) 390.

[5] K. Schmid, J. Roth, J. Nucl. Mater. 313-316 (2003) 302.

[6] K. Schmid, M. Baldwin, R. Doerner, J. Nucl. Mater. 348 (2006) 294.

[7] E. D. de Rooij, U. von Toussaint, A. W. Kleyn, W. J. Goedheer, Phys. Chem. Chem. Phys. 11 (2009) 9823.

[8] K. Krieger, et. al., J. Nucl. Mat. 390-91 (2009) 110.

[9] M. Reinelt, K. Krieger, S. Lisgo, K. Schmid, S. Brezinsek, Interpretation of be migration studies at jet and validation of an integrated numerical model for plasma impurity transport and wall composition dynamics, This conference.

[10] K. Schmid, M. J. Baldwin, R. P. D. D. Nishijima, Nuclear Technology 159, No. 3 (2007) 238.

[11] K. Schmid, Nuclear Fusion 48 (2008) 105004.

[12] C. Garcia-Rosales, W. Eckstein, J. Roth, J. Nucl. Mater. 218 (1994) 8.

[13] J. Bohdansky, Nucl. Instrum. and Meth. B 2 (1984) 587.

[14] W. Möller, W. E. und J. P. Biersack, Computer Physics Communications 51 No. 8 (1988) 355 .

[15] M. Balden, E. D. Pardo, I. Quintana, B. Cieciwa, J. Roth, J. Nucl. Mat. 337 (2005) 980.

[16] J. Brooks, J. Allain, J. Nucl. Mater. 390-391 (2009) 123.

[17] A. Kukushkin, H. Pacher, G. Janeschitz, A. Loarte, D. C. et al., Nucl. Fus. 42 (2002) 187.

[18] K. Schmid, M. Baldwin, R. Doerner, J. Nucl. Mat. 337-339 (2005) 862.

[19] C. Linsmeier, M. Reinelt, K. Schmid, Surface chemistry of first wall materials - from fundamental data to modeling, This conference. 


\section{Figure captions}

Fig. 1

As an example for the re-distribution of eroded material, the charge state integrated Be re-distribution matrix is shown.

Fig. 2

As an example the fit quality of the scale model for the sputter and reflection yields the sputter yield of Be by D and the reflection yield of Be are compared to the TRIDYN calculations.

Fig. 3

Time evolution of the average surface concentration at the inner divertor strike point area 


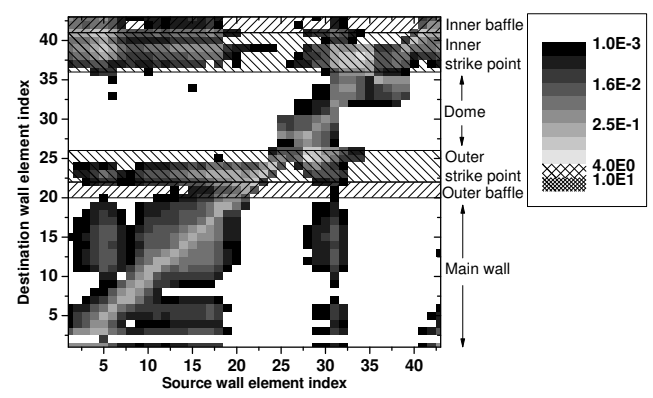

Fig. 1.

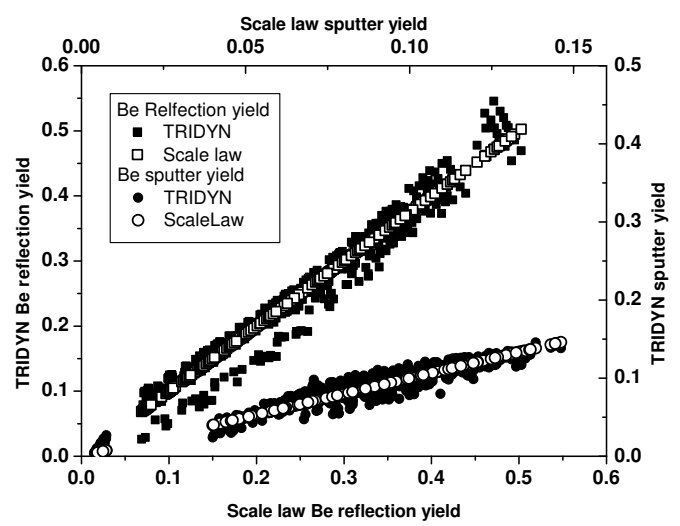

Fig. 2.

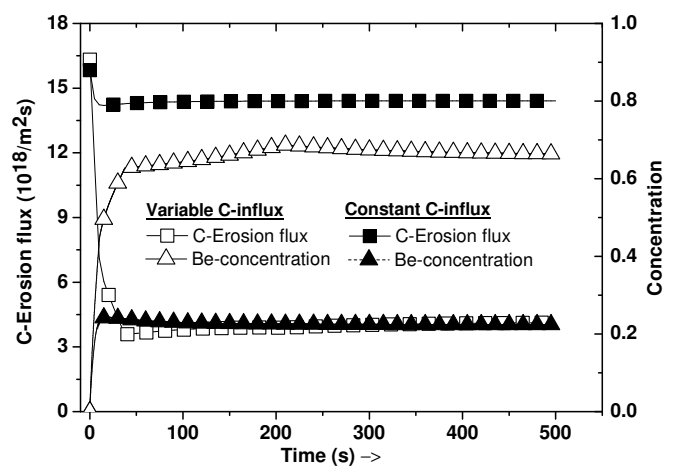

Fig. 3. 